\title{
Article
}

\section{Generation of Surface Energy Patterns by Single Pulse Laser Interference on Self-Assembled Monolayers}

T. Geldhauser, P. Leiderer, J. Boneberg, S. Walheim, and Th. Schimmel Langmuir, 2008, 24 (22), 13155-13160 • DOI: 10.1021/la801812j • Publication Date (Web): 25 October 2008

Downloaded from http://pubs.acs.org on January 28, 2009
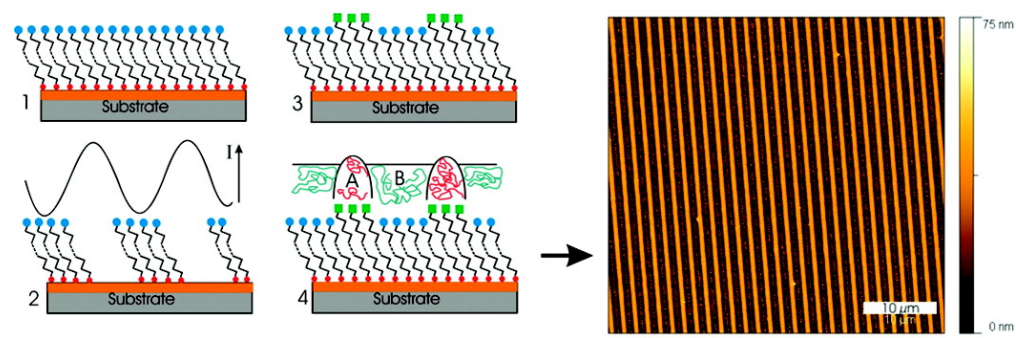

\section{More About This Article}

Additional resources and features associated with this article are available within the HTML version:

- Supporting Information

- Access to high resolution figures

- $\quad$ Links to articles and content related to this article

- Copyright permission to reproduce figures and/or text from this article

\section{View the Full Text HTML}




\title{
Generation of Surface Energy Patterns by Single Pulse Laser Interference on Self-Assembled Monolayers
}

\author{
T. Geldhauser,* P. Leiderer, and J. Boneberg \\ University of Konstanz, Universitätsstr. 10, 78457 Konstanz, Germany
}

S. Walheim*

Institute of Nanotechnology (INT), Forschungszentrum Karlsruhe, D-76021 Karlsruhe, Germany

Th. Schimmel

\begin{abstract}
Institute of Applied Physics, Center for Functional Nanostructures (CFN), Universität Karlsruhe, D-76128 Karlsruhe, Germany, and Institute of Nanotechnology (INT), Forschungszentrum Karlsruhe, D-76021 Karlsruhe, Germany
\end{abstract}

Received June 10, 2008. Revised Manuscript Received September 12, 2008

\begin{abstract}
Single pulse laser interference lithography is used to structure self-assembled monolayers of thiols on gold. This structuring process is investigated by attenuated total reflection measurements, and a demixing process of a binary polymer blend is used to visualize the produced surface energy pattern. The lithography can be realized with different wavelengths $(266,532$, and $1064 \mathrm{~nm})$ which shows that the structuring is a thermal process. As a first demonstration of this process, structures down to $800 \mathrm{~nm}$ period and $300 \mathrm{~nm}$ width are fabricated.
\end{abstract}

In many areas of physics, biology, and chemistry, periodic structures are used. This covers, among others, photonic crystals, ${ }^{1}$ cell adhesion studies on patterned substrates, ${ }^{2}$ wettability of surfaces, ${ }^{3}$ and magnetic data storage. ${ }^{4,5}$ The mechanisms to generate these structures are manifold. Numerous methods have been implemented to generate periodic structures by the usual (top-down) approach, of lithography, where a radiation-sensitive polymer resist layer gets locally destroyed by UV-light or an electron beam. An alternative (bottom-up) approach is to generate a surface energy pattern, on which then an unconventional resist layer assembles. Böltau et al. ${ }^{6}$ have demonstrated phase separation of polymers on chemical patterns fabricated by microcontact printing.

Furthermore molecular layers can be structured by laser light, here usually UV-light is used to photochemically desorb the molecules, as in scanning near field lithography. ${ }^{7}$ Peters et al. ${ }^{8}$ have demonstrated the patterning of octadecyltrichlorosilane (OTS) by XUV light from a synchrotron source, with a Loyds mirror. Subsequent demixing of diblock-co-polymers on a generated surface energy pattern have led to periods down to 50 nm. ${ }^{9,10}$ This shows that this approach of surface energy pattern replication into a polymer layer works even for that lengthscale.

* tobias.geldhauser@uni-konstanz.de; stefan.walheim@int.fzk.de.

(1) Joannopoulos, J. D.; Villeneuve, P. R.; Fan, S. Nature 1997, 386(6621), $143-149$.

(2) Chen, C. S.; Mrksich, M.; Huang, S.; Whitesides, G. M.; Ingber, D. E. Science 1997, 276(5317), 1425-1428.

(3) Zhao, Y.; Lu, Q.; Li, M.; Li, X. Langmuir 2007, 23(11), 6212-6217.

(4) Tselev, A. E.; Polushkin, N. I.; Verevkin, Y. K.; Gorbunov, A. A.; Petryakov, V. N.; Pompe, W. Appl. Phys. A: Mater. Sci. Process. 1999, 69, 819-822.

(5) Albrecht, M.; Hu, G.; Guhr, I. L.; Ulbrich, T. C.; Boneberg, J.; Leiderer, P.; Schatz, G. Nat. Mater. 2005, 4(3), 203-206.

(6) Böltau, M.; Walheim, S.; Mlynek, J.; Krausch, G.; Steiner, U. Nature 1998, 391, 877 .

(7) Sun, S.; Leggett, G. J. Nano Lett. 2002, 2(11), 1223-1227.

(8) Peters, R. D.; Yang, X. M.; Wang, Q.; Pablo, J. J.; Nealey, P. F. J. Vac. Sci. Technol., Sect. B 2000, 18, 3530-3534.

(9) Stoykovich, M. P.; Muller, M.; Kim, S. O.; Solak, H. H.; Edwards, E. W.; de Pablo, J. J.; Nealey, P. F. Science 2005, 308(5727), 1442-1446.

(10) Yang, X. M.; Peters, R. D.; Nealey, P. F.; Solak, H. H.; Cerrina, F. Macromolecules 2000, 33(26), 9575-9582.
With a tabletop laser source, Chen et al. ${ }^{11}$ have periodically patterned a polymer resist, using the third harmonic of a $\mathrm{Nd}$ : YAG laser. By direct laser writing with a focused Ar-ion laser, ${ }^{12,13}$ Shadnam et al. ${ }^{14,15}$ structured thiols in the vis range of laser light by thermal desorption.

In this paper, we demonstrate a new parallel patterning process by single pulse laser interference lithography (SPLIL) with vis light relying on fast thermal desorption of a self-assembled monolayer (SAM) on the nanosecond scale, leading to sharp structures due to the short thermal desorption length. The removal of the monolayer by the laser treatment is monitored by surface plasmon resonance (SPR) measurements. The generation of an effective surface energy pattern by LIL is proved by its ability to control the lateral demixing of a polymer blend on these laserpatterned substrates.

There are several advantages of nanosecond-pulsed laser interference. In contrast to direct laser writing, ${ }^{12-15}$ it is a parallel method which allows one to structure square millimeters in a single shot. Due to the nanosecond pulse length, it is not necessary to stabilize the experiment against lateral drifts. The coherent laser beam in the $\mathrm{UV}-$ vis range makes it possible to use standard laser optics for the patterning process, and the period can be varied over a wide range by changing the angles of the incident laser beams. For more complicated structures, holographic techniques can be applied. In order to understand the mechanisms of our new lithography technique, the properties of our ultrathin resist layers have to be addressed. The self-assembled monolayer on the used gold substrate consists of alkanethiols which form a dense monolayer by covalent bonding between the sulfur endgroup

(11) Chen, X.; Zaidi, S. H.; Brueck, S. R. J.; Devine, D. J. J. Vacuum Sci. Technol. B: Microelectr. Nanometer Struct. 1996, 14(5), 3339-3349.

(12) Balgar, T.; Franzka, S.; Hartmann, N.; Hasselbrink, E. Langmuir 2004, 20(9), 3525-3527.

(13) Balgar, T.; Franzka, S.; Hasselbrink, E.; Hartmann, N. Appl. Phys. A: Mater. Sci. Process. 2006, 82(1), 15-18.

(14) Shadnam, M. R.; Amirfazli, A. Chem. Commun. 2005, 38, 4869-4871.

(15) Shadnam, M. R.; Kirkwood, S. E.; Fedosejevs, R.; Amirfazli, A. Langmuir 2004, 20(7), 2667-2676. 
and gold ${ }^{16}$ or other coinage metals. ${ }^{17}$ In this monolayer, the molecules form a $(\sqrt{ } 3 \times \sqrt{3}) R 30^{\circ}$ honeycomb structure on the surface. $^{18,16,19}$

The structuring of a thiol SAM on a gold surface by light in the UV range can be achieved by either a photochemical or a photothermal process. ${ }^{35}$ In the photochemical process, the whole molecule is desorbed or bonds between $\mathrm{C}-\mathrm{C}$ and $\mathrm{C}-\mathrm{S}$ are broken by photoscissoring, ${ }^{20}$ the bond breakage can also be relevant in the photothermal regime. ${ }^{21}$ Another way is the photothermal desorption, which turned out to be the main path of structuring in our approach. Thermal desorption studies (TDS) show that the critical temperature for the desorption of thiols on gold depends on the ambient conditions but has nearly no dependence on the chainlength. ${ }^{22}$ Scanning tunneling microscopy (STM) measurements in ultrahigh vacuum (UHV) from Camillone et al., ${ }^{23}$ and TDS measurements by Nuzzo et al. ${ }^{18}$ report a desorption temperature of $575 \mathrm{~K}$ for decanethiol in vacuum at a heating rate of $1 \mathrm{~K} / \mathrm{s}$ on a $\mathrm{Au}(111)$ surface. Yang et al. ${ }^{21}$ have shown by high resolution X-ray photoelectron spectroscopy (XPS) measurements in vacuum that $\mathrm{C}-\mathrm{S}$ cleavage at $415 \mathrm{~K}$ lead to vacuum desorption of the alkyl chain, whereby residual atomic sulfur was measured on the gold surface. The authors state that in their experiment the sulfur desorbs separately at higher temperatures. Shadnam et al.$^{14}$ report desorption experiments in air with a temperature of over $383 \mathrm{~K}$ for the decomposition of thiol and a binding energy of $30.5 \mathrm{kcal} / \mathrm{mol}$, which is in good agreement to the chemical binding energy of $1.28 \mathrm{eV}$ found by Schreiber et al. ${ }^{22}$ Burgess et al. have shown that at higher rates higher desorption temperatures can be found. ${ }^{24}$ In our case, extremely high heating rates are realized. With a $13 \mathrm{~ns}$ laser pulse, a heating rate of $10^{8}-10^{11} \mathrm{~K} / \mathrm{s}$ is expected; therefore, in our case the effective desorption temperature will be increased, so that in the extremely short heating time a significant number of molecules desorb. Considering the data above, a chemical modification as well as a complete desorption of thiol molecules or both could result from the interaction of the sample with an intense nanosecond pulse.

In order to quantify the destruction of SAMs after the laser treatment, we make use of the fact that thiol SAMs grow on a thin gold layer. This makes it possible to use surface plasmon resonance (SPR) as a very sensitive tool to investigate changes on the surface of the metal film. Monitoring of structural changes in a molecular film have been demonstrated e.g. by Damos et al. ${ }^{25}$ and for mercaptoundecanol by Pettit et al. ${ }^{26}$

Since the goal of this study is the generation of surface energy patterns by laterally controlled desorption of SAM molecules, we have to prove this property of the patterns derived from our lithography by a probe which is sensitive to surface energy fluctuations. A process which reacts strongly on surface energy is the phase separation of a polymer blend during the film

(16) Molina, L. M.; Hammer, B. Chem. Phys. Lett. 2002, 360(3-4), 264-271. (17) Love, J. C.; Wolfe, D. B.; Haasch, R.; Chabinyc, M. L.; Paul, K. E.; Whitesides, G. M.; Nuzzo, R. G. J. Am. Chem. Soc 2003, 125(9), 2597-2609.

(18) Nuzzo, R. G.; Dubois, L. H.; Allara, D. L. J. Am. Chem. Soc. 1990, $112(2), 558-569$.

(19) Ulman, A. Chem. Rev. 1996, 96(4), 1533-1554.

(20) Love, J. C.; Estroff, L. A.; Kriebel, J. K.; Nuzzo, R. G.; Whitesides, G. M. Chem. Rev. 2005, 105(4), 1103-1170.

(21) Yang, Y. W.; Fan, L. J. Langmuir 2002, 18(4), 1157-1164.

(22) Schreiber, F.; Eberhardt, A.; Leung, T. Y. B.; Schwartz, P.; Wetterer, S. M.; Lavrich, D. J.; Berman, L.; Fenter, P.; Eisenberger, P.; Scoles, G. Phys. Rev. B 1998, 57(19), 12476-12481.

(23) Camillone, N., III; Eisenberger, P.; Leung, T. Y. B.; Schwartz, P.; Scoles, G.; Poirier, G. E.; Tarlov, M. J. J. Chem. Phys. 1994, 101, 11031.

(24) Burgess, D., Jr.; Stair, P. C.; Weitz, E. J. Vacuum Sci. Technol. A: Vacuum, Surf. Films 1986, 4, 1362.

(25) Damos, F. S.; Luz, R. C. S.; Kubota, L. T. Langmuir 2005, 21(2), 602609.

(26) Pettit, C. M.; Roy, D. The Analyst 2007, 132(6), 524-535.


Figure 1. Schematic of the nanostructuring process. After the hydroxyl (blue circles) terminated thiols (red circles) generate a SAM of 11-MUD (1), the molecules are partially desorbed by LIL (2) and the free gold surface is reoccupied by the methyl (green squares) terminated ODT (3). The patterned surface leads to a directed phase separation of the polymer solution (4) of two immiscible polymers A and B.

formation by spin coating. ${ }^{27}$ During the film formation, the (common) solvent evaporates and two solvent-rich polymer phases develop, from which one (e.g., the more polar polymer component) is usually attracted by a polar substrate, whereby the more unpolar component is usually attracted by the solution-air interface. On polar stripes, it is possible to direct this structure formation into a well-ordered lateral layout which is given by the intended surface energy pattern. ${ }^{6,27-30}$ We use a wellinvestigated polymer blend system, which strongly reacts on a surface energy pattern by nearly perfect replication. ${ }^{6}$ The polymer structures-produced by a fast and simple spincoating processthus give us a versatile image of the surface energy pattern generated by our laser interference lithography. At the same time, we are able to amplify the monomolecular patterns-which themselves are only $2 \mathrm{~nm}$ in height-by a factor of 30 in thickness. The result is a laterally organized polymer film with a thickness of about $60 \mathrm{~nm}$. By using a selective solvent, one of the polymers can be dissolved and the remaining polymer component can be used as a resist layer for further processing like, e.g., reactive ion etching.

\section{Experimental Section}

Formation and Structuring of the Monolayer. The substrate in our nanostructuring process was a p-doped (111) oriented silicon wafer. We evaporated a $1 \mathrm{~nm}$ chromium adhesive layer and a 40 nm gold layer on top. In the next step, the self-assembled monolayer (SAM) of 11-mercapto-undecanol (MUD), a hydroxyl terminated thiol, is formed by submersing the gold surface for $10 \mathrm{~min}$ in ethanol with 5 mmol MUD (Figure 1) to ensure that a full monolayer of the SAM is formed. ${ }^{41}$ For laser interference lithography, we used an injection seeded Nd:YAG laser with its fundamental (1064 nm), second $(532 \mathrm{~nm})$, and fourth $(266 \mathrm{~nm})$ harmonic and pulsewidths around $13 \mathrm{~ns}$. The beam was split in two equally intense beams which are rejoined, after traveling the same length, on the SAM-gold surface under a certain angle. This leads to a periodic modulated intensity by interference of the two beams with a periodicity determined by the fraction of the wavevector $k=2 \pi / \lambda$ of the two beams in the $x-y$ plane on the substrate as

$$
I(x)=2 I_{0}[1+\cos (2 x k \sin \theta)]
$$

with the angle $2 \theta$ between the incident beams. Typical values in the experiment shown here were periods of 1.8 down to $0.8 \mu \mathrm{m}$. The

(27) Cyganik, P.; Bernasik, A.; Budkowski, A.; Bergues, B.; Kowalski, K.; Rysz, J.; Lekki, J.; Lekka, M.; Postawa, Z. Vacuum 2001, 63(1-2), 307-313.

(28) Rockford, L.; Liu, Y.; Mansky, P.; Russell, T. P.; Yoon, M.; Mochrie, S. G. J. Phys. Rev. Lett. 1999, 82(12), 2602-2605.

(29) Karim, A.; Douglas, J. F.; Lee, B. P.; Glotzer, S. C.; Rogers, J. A.; Jackman, R. J.; Amis, E. J.; Whitesides, G. M. Phys. Rev. E 1998, 57(6), 6273-6276. 


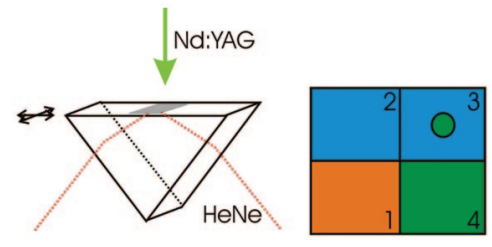

Figure 2. (left) Prism for the SPR measurements mounted on a translation stage, to enable scans over certain areas. If needed a Nd:YAG is available for in situ desorption. (right) Segmented substrate with pure gold (1), 11-MUD (2, blue), ODT (4, green), and the mixed area (3).

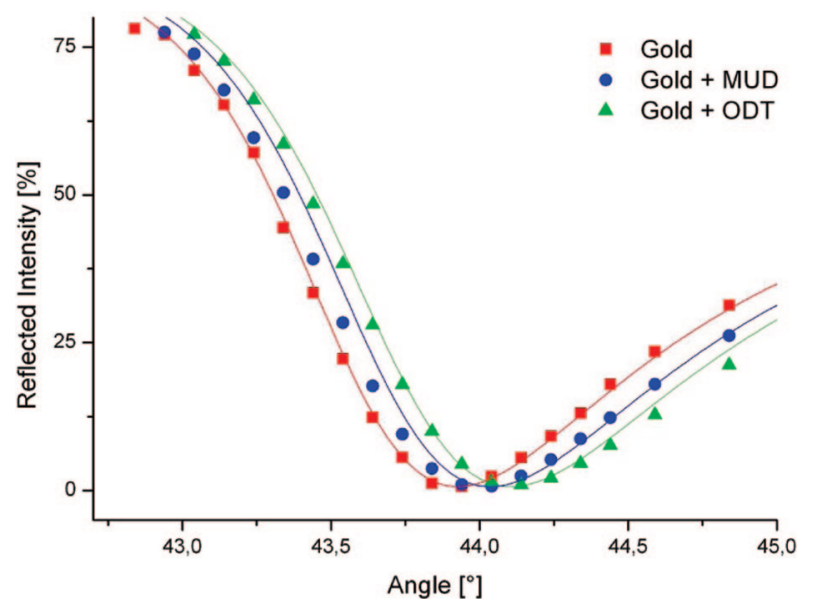

Figure 3. Measurement of the reflectivity $R$ by ATR of the pure gold film (red, square), gold with 11-MUD (blue, dots), and gold with ODT (green, triangles). The angle of resonance shifts with increasing length of the molecules.

Table 1. Characteristics of the Used Polymers

\begin{tabular}{ccc}
\hline polymer & $M_{\mathrm{w}}\left(10^{3}\right)[\mathrm{D}]$ & $M_{\mathrm{n}}\left(10^{3}\right)[\mathrm{D}]$ \\
\hline PS & 9.58 & 9.32 \\
PVP & 11.1 & 10.7
\end{tabular}

periodic intensity distribution is partially absorbed in the gold and leads to a periodic temperature profile (Figures 1 and 2) and as a consequence to a desorption of the SAM in the high temperature areas.

In the LIL experiments on gold on silicon, we used peak fluences of $28.5 \mathrm{~mJ} / \mathrm{cm}^{2}$ for each Gaussian beam with $5.2 \mathrm{~mm}$ full width at half maximum (fwhm). With these energies, the structuring process was done by SPLIL.

In the next step, the substrate is submerged in a $3 \mathrm{mmol}$ solution of 1-octadecanethol (ODT) with ethanol for $2 \mathrm{~min}$, to occupy the free gold surface with a methyl terminated SAM (Figures 1 and 3), and a chemically patterned surface with different surface energies is formed.

Polymer Phase Separation. As the phase-separating polymer solution, we spin-cast a polymer blend consisting of a 0.5 wt \% solution of poly-2-vinylpyridine (PVP) and polystyrene (PS) (Table 1) in tetrahydrofuran at $3000 \mathrm{rpm}$ at a relative humidity $30 \%$ onto the chemically patterned substrate. There, the polymers are phase separated (Figures 1 and 4). To reveal the generated pattern, we washed the sample in cyclohexane, as selective solvent for polystyrene and only the PVP pattern remains on the substrate.

Attenuated Total Reflection Measurements. For our attenuated total reflection (ATR) studies, we used a setup illustrated in Figure 2. A helium-neon laser $(\mathrm{HeNe})$ with a diameter of $0.35 \mathrm{~mm}$ is probing the angle-dependent reflection of a glass substrate with 1 $\mathrm{nm}$ of chromium and $43 \mathrm{~nm}$ of gold on top of a glass prism. An $x y$-translation stage is used to scan different areas of the substrate (Figure 2 right). We prepared four different sections on one substrate to ensure that the substrate conditions were identical for all ATR

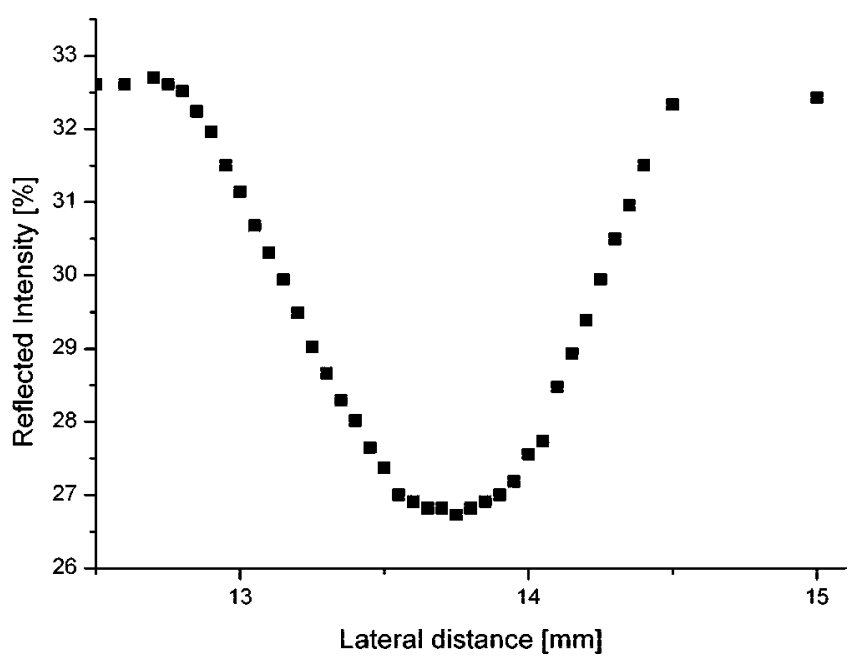

Figure 4. ATR scan over the desorbed MUD SAM after irradiation with a Gaussian laser beam of $88 \mathrm{~mJ} / \mathrm{cm}^{2}$ and a fwhm of $5.2 \mathrm{~mm}$.

measurements. For that purpose first, the upper half of the substrate (area 2 and 3) was immersed into MUD solution. Then in area 3, a Nd:YAG laser desorption experiment with a Gaussian beam of 5.2 $\mathrm{mm}$ fwhm was performed. Finally the substrate was turned by $90^{\circ}$ and areas 3 and 4 were immersed into ODT. Therefore the pattern consists of the bare Au layer in area 1, and areas 2 and 4 of the gold surface are occupied by 11-MUD and ODT, respectively. On the third segment, a circular spot ODT surrounded by 11-MUD has been generated.

For the simulation of the surface plasmon resonance, we used the transfer matrix method. ${ }^{31-33}$

\section{Results}

For the later investigation of the lateral structuring process by LIL, we first characterized the desorption process on the macroscale, where we used the attenuated total reflection measurement. The ATR spectra of the substrate is shown in Figure 3 (red squares). As the surface is covered with a dense molecule layer, the plasmon resonance shifts to higher angles (Figure 3). In the case of the $1.1 \mathrm{~nm}$ long 11-MUD, a shift of $0.11^{\circ}$ and for ODT (length $1.8 \mathrm{~nm}$ ) of $0.21^{\circ}$ is measured. This coincides very well with the simulated ATR spectra of the molecules with a dielectric constant of $\varepsilon_{1}=2.1,{ }^{25,34}$ considering the tilt of the molecules. ${ }^{16,18,19}$ When the substrate heats up due to the interaction with the laser of $532 \mathrm{~nm}$ wavelength and a Gaussian profile (Figures 2 right and 3) of $5.2 \mathrm{~mm}$ fwhm, the molecules are desorbed at a high enough fluence $\left(88 \mathrm{~mJ} / \mathrm{cm}^{2}\right)$ as can be seen in Figure 4 in a scan over the irradiated area. For this scan, we used an angle of $43.5^{\circ}$, corresponding to a reflectivity of $26,4 \%$ and $32,6 \%$ for gold and MUD. On the outer areas, the intact MUD layer can be seen, whereas the reflectivity in the center, where the temperature was highest, corresponds to a complete desorption of the monolayer, as it relates to the reflectivity of the free gold surface. Between these spots, the reflectivity gradually decreases. On another spot, we immersed the substrate in ODT to recover the free gold substrate after

(30) Andrew, P.; Huck, W. T. S. Soft Matter 2007, 3(2), 230-237.

(31) Azzam, R. M. A.; Bashara, N. M. Ellipsometry and polatized light; NorthHolland: Amsterdam, 1992.

(32) Armstrong, R. L.; King, J. D. The electromagnetic interaction; PrenticeHall: Engelwood Cliffs, 1973.

(33) Holler, G.; Albrecht, U.; Herminghaus, S.; Leiderer, P. Appl. Phys. Lett. 1993, 62(22), 2877.

(34) Jordon, C. E.; Frey, B. L.; Kornguth, S.; Corn, R. M. Langmuir 1994, 10(10), 3642-3648.

(35) Baeuerle, D. Laserprocessing and Chemistry; Springer: New York, 2000. 




Figure 5. ATR scan after the MUD layer has been desorbed at a fluence of $100 \mathrm{~mJ} / \mathrm{cm}^{2}$ and refilled with ODT.
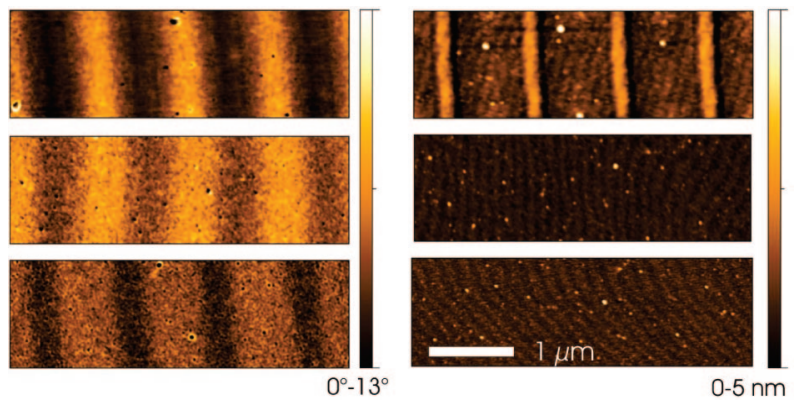

Figure 6. AFM image of the topography (right) and the phase contrast (left) of the MUD and ODT stripes generated by UV LIL. The intensity is decreasing from the upper to the lower pictures.

desorption of the 11-MUD (Figure 5). With a fixed angle of $43.7^{\circ}$, the reflected intensities of MUD and ODT are $9.5 \%$ and $18.9 \%$.

In Figure 5, the ATR signal in the center corresponds to a complete layer of a dense monolayer of ODT molecules. Between the different dense monolayers is a transition region $0.75 \mathrm{~mm}$ in width. As the scans are from different experiments, a variation in the beam profile can be seen. For a comparison, we measured the impact of the test laser profile. We identified the fwhm of the beam to $0.35 \mathrm{~mm}$ with a knife-edge scan.

After we have shown that-with sufficient laser intensitymolecules of the SAM can be removed and replaced, the next step is to use the interference pattern to laterally structure the substrate with alternating MUD and ODT stripes.

Although the MUD and ODT molecules differ in length, the direct topographic imaging of the molecule structures is not possible due to the roughness of the gold surface. In Figure 6, the topographic and the simultaneously gathered phase contrast images by SPLIL with $266 \mathrm{~nm}$ wavelength and a period of $1 \mu \mathrm{m}$ are shown by atomic force microscopy (AFM). Here, the darker areas correspond to the modified SAM of MUD and a partial or full ODT monolayer, depending on the surface temperature, as shown in the ATR measurements. Scans were performed starting from the center of the Gaussian beam with a fluence higher than $120 \mathrm{~mJ} / \mathrm{cm}^{2}$ (Figure 6, top), where the gold surface is molten, to areas of lower fluence (Figure 6, bottom). As to be expected, the line width of the desorbed and later refilled structures decreases with decreasing fluence.

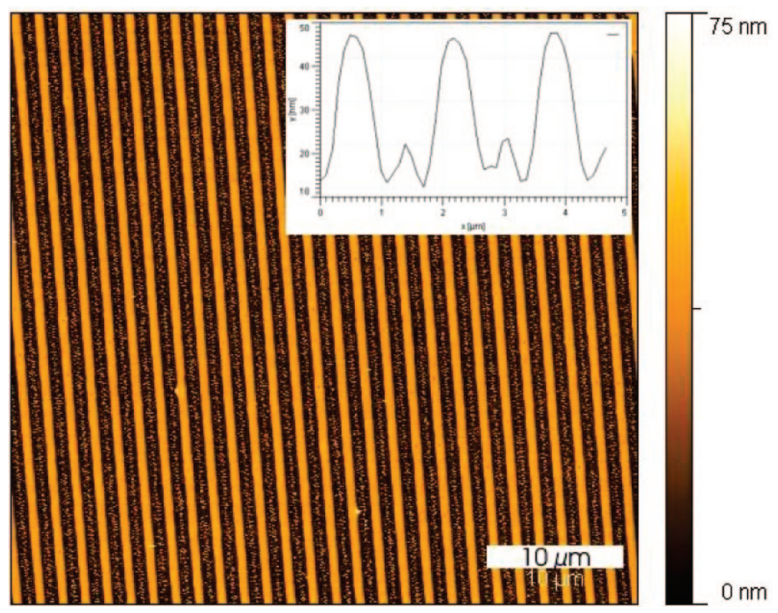

Figure 7. AFM image of PVP structures generated by LIL with UV $(266 \mathrm{~nm})$ light and phase separation of a binary polymer blend. The second polymer (PS) has been dissolved by cyclohexane.
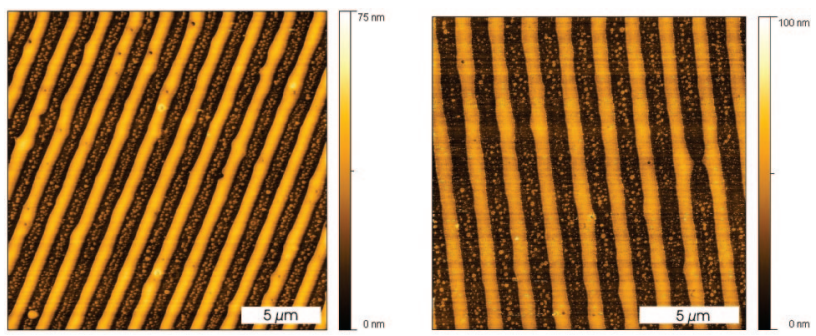

Figure 8. AFM images of PVP patterns of $1.8 \mu \mathrm{m}$ period, after directed demixing of the PS-PVP solution on the chemical pattern, generated at the wavelengths 1064 (left) and $532 \mathrm{~nm}$ (right). The PS has been removed by cyclohexane.

In order to increase the visibility of the surface energy structures, and as a demonstration of its application, we used the demixing of the PS/PVP solution as a imaging technique for the successful patterning of the gold surface. Since MUD provides a highly polar surface, the expected ODT/MUD patterns have a rather high contrast in surface energy. In Figure 7, the AFM image of the result of a spin coating process of PS and PVP dissolved in THF on such a laser-patterned substrate is shown after the PS has been removed by cyclohexane. The gold substrate was first coated with MUD, and after single pulse laser treatment, the ODT was filled in. The polymer structure is perfectly aligned along the desired line pattern. The line pattern has a periodicity of $1.8 \mu \mathrm{m}$ and was produced by using the $266 \mathrm{~nm}$ light.

Since we used UV light, the desorption of the SAM could either be photochemical by bond breaking or photothermal. In order to evaluate the main patterning mechanism for the LIL, we used in the next step the two other wavelengths of the Nd:YAG laser to pattern the SAM. For a better comparison of the results, a periodicity of $1.8 \mu \mathrm{m}$ and a equivalent fluence of each pulse of $28.5 \pm 2 \mathrm{~mJ} / \mathrm{cm}^{2}$ for $532 \mathrm{~nm}$ wavelength on the silicon substrate was chosen in all three cases. This corresponds to a fluence of $105.6 \pm 8 \mathrm{~mJ} / \mathrm{cm}^{2}$ in the maxima of the interference pattern. The resulting patterns of the PVP are shown in Figure 7 for $266 \mathrm{~nm}$ and Figure 8 for 532 and $1064 \mathrm{~nm}$.

The generated structures at $1.8 \mu \mathrm{m}$ periodicity have a width of $600 \mathrm{~nm}$ and a height of $40-50 \mathrm{~nm}$. With another configuration, patterns down to a period of $800 \mathrm{~nm}$ could be realized (Figure 9). 


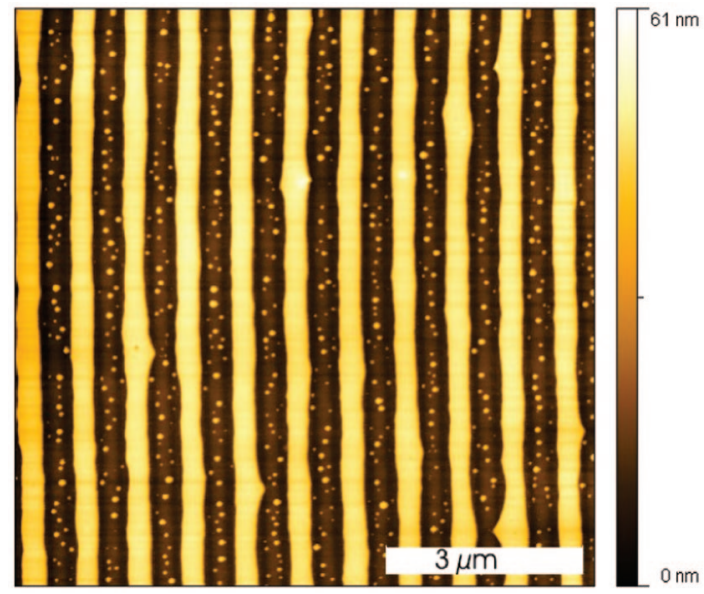

Figure 9. AFM image of the generated PVP structures by SPLIL with UV $(266 \mathrm{~nm})$ and a period of $800 \mathrm{~nm}$.

\section{Discussion}

In Figures 7-9, small droplets between the polymer lines can be seen, this can be traced to PVP which did not reach the attractive areas during the demixing process. Similar structures between the two lines have been observed. ${ }^{6,27}$

Comparing the PVP pattern generated by the structuring process with UV light (4.64 eV, Figure 7), visible (2.32 eV), and IR light (1.16 eV, Figure 8), no difference due to the photon energy can be identified. This observation is a strong indication that the main patterning process of the SAM is independent of the photon energy and thus the thiol molecules are removed by photothermal desorption. In case of the UV light, the photochemical removal of the thiol might occur additionally, but the photothermal process itself is sufficient. ${ }^{35}$

In order to get a deeper understanding of the desorption mechanism, we used heat flow simulations combined with the Eyring equation to simulate the change in coverage of the molecule layer.

With a Gaussian beam diameter on the order of millimeters, as used in the ATR experiment, we can use one-dimensional numerical heat flow simulations to get the temporal temperature profile on the gold surface, ${ }^{36,37}$ and with this, the coverage $C(T, t)$ of the molecules on the surface can be calculated with the Eyring equation $^{14,38}$

$$
C(T, t) \sim \exp \left(-\frac{k_{\mathrm{b}}}{h} T t \exp \left(-\frac{\Delta G}{R T}\right)\right)
$$

As mentioned earlier, the Gibbs free energy for desorption $\Delta G$ has been quantified to $30.5 \mathrm{kcal} / \mathrm{mol} .{ }^{14,22}$ These calculations suggest a full desorption of the molecules at a wavelength of 532 $\mathrm{nm}$ and the temporal profile of $13 \mathrm{~ns}$ fwhm at a fluence of 87 $\mathrm{mJ} / \mathrm{cm}^{2}$ (Figure 10 top).

Furthermore, the ATR measurements suggest a transition region where only part of the molecules are desorbed. The knife-edge scan has shown that the transition region observed in Figure 4 is not due to the laser beam profile but is a result of the partial desorption of the monolayer. This agrees completely with the heat flow simulations at slightly lower energy densities $(85 \mathrm{~mJ} /$ $\mathrm{cm}^{2}$, Figure 10, bottom), where only a partial desorption is

(36) Grigoropoulos, C. P.; Park, H. K.; Xu, X. Int. J. Heat Mass Transf. 1993, 36(4), 919-924.

(37) Poate, J. M. Laser annealing of semiconductors: Academic Press: New York, 1982.

(38) Lowry, T. H.; Richardson, K. S. Mechanism and Theory in Organic Chemistry; Harper \& Row: New York, 1987.


Figure 10. Calculated surface temperature (red) and the changing coverage of the molecules (black) due to the incident Nd:YAG laser pulse with 87 (top) and $85 \mathrm{~mJ} / \mathrm{cm}^{2}$ (down). The dashed curve illustrates the Nd:YAG pulse.

observed. Experimentally, we find a high enough surface temperature in the center region to completely desorb the molecules (Figure 4). This complete removal allows to add another dense molecule layer in this area as indicated by ATR measurements (Figure 5), whereas in the transition region only a part of the molecules can be exchanged. The rather large difference in the desorption for a small difference in the laser fluence and the resulting peak temperature of the temporal profile is a result of the exponential behavior of the Eyring equation.

By comparing the lateral change in fluence of the Gaussian beam used in Figure 4 with the calculated coverage and the corresponding reflectivity in the ATR measurements, a very good agreement of the calculations and the experiments can be seen. This applies analogous for the measurements seen in Figure 5.

For the single pulse patterning on the silicon substrate, a higher fluence compared to glass is needed as the heat diffusion in silicon is faster than that in glass; nevertheless in both cases, the desorption of the molecules sets in before the gold is molten. Furthermore, the gold is not an ideal substrate as the desorption energy $\left(87 \mathrm{~mJ} / \mathrm{cm}^{2}\right)$ is not far away from the melting threshold for the gold surface $\left(115 \mathrm{~mJ} / \mathrm{cm}^{2}\right){ }^{40}$

The thermal desorption process was finally used for lateral structuring of the surface; here, the thermal structuring of the molecules relies on the localization of the heat in the desired

(39) Kaganovskii, Y.; Vladomirsky, H.; Rosenbluh, M. J. Appl. Phys. 2006, $100,044317$. 149.

40) Boneberg, J.; Bischof, J.; Leiderer, P. Opt. Commun. 2000, 174, 145-

(41) Bain, C. D.; Troughton, E. B.; Tao, Y. T.; Evall, J.; Whitesides, G. M.; Nuzzo, R. G. J. Am. Chem. Soc. 1989, 111, 321-335. 
area. As two-dimensional heat flow calculations show that for interference illumination with $1 \mu \mathrm{m}$ period the lateral temperature profile disappears at the end of the laser pulse due to heat diffusion, ${ }^{39}$ we were expecting not to reach lateral structuring below $1 \mu \mathrm{m}$. The heat diffusion length

$$
d_{\text {heat }}=\sqrt{\kappa_{\mathrm{f}} \tau}
$$

for a pulse of $\tau=13 \mathrm{~ns}$ on a gold substrate with the heat diffusivity $\kappa_{\mathrm{f}}$ of $1.26 \times 10^{-4} \mathrm{~m}^{2} / \mathrm{s}$ is $1.28 \mu \mathrm{m} .{ }^{39}$ Nevertheless, the experiment showed that much smaller structures are achievable (Figure 9). The reason for this is the thermal desorption process, where the highest temperatures are dominating and even slightly lower temperatures (Figure 10), as in the case of the flattened temperature profile, ${ }^{39}$ are insufficient to completely desorb the molecules. Obviously the induced surface energy contrast is high enough to direct the different polymers on the desired pattern.

In contrast to the ATR measurements, no clear conclusions on the extend of the molecule desorption can be drawn from the phase contrast images in Figure 6, but a clear modification and contrast in the surface energy depending on the laser fluence is visible.

\section{Conclusion}

We have shown a new and versatile method to generate chemically patterned surfaces in a parallel process. The pattern- induced demixing of a polymer blend has been used to amplify and visualize the SAM structures by AFM. ATR spectra show quantitatively the desorption by laser treatment and a successful redeposition of new SAM molecules. We found strong indications that the main path of structuring is by photothermal desorption of the molecules. In this case, the ATR spectra showed that the desorption process, as expected, does not have a sharp intensity threshold, but a transition to full desorption can be seen. For the thiols, a mixed phase between the MUD and ODT molecules could be shown in this transition region. One-dimensional heat flux simulations have confirmed these results.

By demixing of a polymer blend, periodic structures $60 \mathrm{~nm}$ in height and $800 \mathrm{~nm}$ in period could be generated by structuring a molecular layer.

This versatile structuring process can be easily transferred onto other substrates and molecules like silanes on silicon.

Acknowledgment. We thank the Kompetenznetz funktionelle Nanostrukturen of the Landesstiftung Baden-Wuerttemberg (Project B4) and the Deutsche Forschungsgemeinschaft within the DFG-Center for functional Nanostructures (CFN) within Project B1.1.

LA801812J 